\title{
ECS effects on the extinction of a running response following CRF or VR training
}

\author{
A. GRANT YOUNG \\ Louisiana State University, Baton Rouge, Louisiana 70803
}

\begin{abstract}
Thirty-two male albino rats were trained to run a straight runway on either a continuous reinforcement schedule (CRF) or a variable ratio (VR) schedule of reinforcement. Following 100 acquisition trials, half of the subjects in each reinforcement group received a single electroconvulsive shock (ECS) while the other half received no treatment. Results of 30 extinction trials showed that, overall, VR-trained subjects ran faster than did CRF-trained subjects; however, those CRF-trained subjects which experienced ECS were not significantly different from VR-trained subjects. The ECS treatment thus eliminated the usual partial reinforcement effect. Results were interpreted in terms of an increased drive or hyperactivity produced by the ECS.
\end{abstract}

When electroconvulsive shock (ECS) is experienced shortly after a learning experience, the result appears to be a retrograde amnesia (RA) for the learning experience (Lewis, 1969). This result is usually explained in terms of a consolidation theory of memory. The idea is that, following a sensory input, a period of time is required for the memory trace to become permanently fixed. This fixation of the memory trace presumably occurs through a physiological consolidation process and if this process is interrupted, for example by ECS, the memory trace does not become fixed and is thus lost (McGaugh, 1966).

A typical procedure for the study of ECS-induced RA is to administer aversive stimulation, such as footshock, following the occurrence of a previously learned response. The aversive stimulation results in a suppression of the response; however, if the aversive stimulus is followed by ECS, response suppression does not occur. thus indicating RA for that one-trial learning experience.

Recent studies, however, have produced findings which suggest that the primary effect of ECS may be one of hyperactivity or increased drive level, indicated by an inflated rate of responding (Dempsey \& Young, 1973; Young \& day, 1971). Young and Costelloe (1974) trained rats to leverpress on either a continuous or partial reinforcement schedule. Following acquisition, half of the subjects in each group received ECS while the other half received sham ECS. All subjects were then extinguished and results showed an inflated response rate in extinction for ECS-treated subjects.

Essentially all of these studies have studied the effect of ECS on a single response-that of leverpressing-and the purpose of this experiment was to examine the effects of ECS on a running

This research was supported in part by a grant from the University Council on Research. LSU. response following continuous and variable ratio reinforcement.

\section{METHOD}

\section{Subjects}

The subjects were 32 naive male albino rats, $175-200 \mathrm{~g}$ in weight at the start of the experiment.

\section{Apparatus}

The apparatus consisted of a straight Plexiglas runway, 4 in. high, $41 / 8 \mathrm{in}$. wide, and $63 \mathrm{in}$. long, including the startbox $(63 / 4 \mathrm{in}$. long) and goalbox (10 in. long). The runway had a grid floor of $1 / 4$-in. brass rods set apart 5/8 in. A foodcup was attached at floor level to the back of the goalbox and guillotine doors separated the startbox and goalbox from the runway. Raising the startbox door started a timer, calibrated to $.1 \mathrm{sec}$, and the timer was automatically stopped when the subject broke a photobeam directed across the runway $1 \mathrm{in}$. from the goalbox entrance.

\section{Procedure}

The subjects were randomly selected from the LSU colony and placed in individual cages on ad-lib food and water for 4 days, after which they were placed on a food deprivation schedule of $10 \mathrm{~g}$ Purina chow every $24 \mathrm{~h}$. Water was available in the cages at all times, and subjects were fed approximately $10 \mathrm{~min}$ after each experimental session.

From Days 5-9, subjects were handled in pairs for 5 min daily, and on Days 10 and 11 were given five direct placements in the goalbox with reinforcement consisting of two 45-mg Noyes food pellets. On Day 12, all subjects were given five alley runs on continuous reinforcement. and on the following day acquisition began.

On the 1st day of acquisition, subjects were divided into two groups. CRF and VR. The subjects in the CRF group received one 45-mg food pellet in the goalbox on each trial, and subjects in the VR group received one 45-mg pellet in the goalbox on a VR-2 schedule. with the restriction that no more than three consecutive runs were either reinforced or nonreinforced.

An acquisition irial consisted of placing the subject in the startbox and raising the door when the subject oriented toward it. The subject was allowed to traverse the runway, and the goalbox door was lowered after the subject entered the goalbox. All subjects were detained in the goalbox for $20 \mathrm{sec}$ on all trials and were then removed to a holding cage for a $20-\mathrm{sec}$ intertrial interval. Acquisition consisted of 10 trials daily and was continued for 10 days.

On the day following acquisition. each group was randomly subdivided, resulting in the following four groups: 
CRF(NT) and VR(NT). The subjects in these two groups were given one nonreinforced runway trial followed by sham ECS, which consisted of attaching microalligator clips to the subject's ears for $1 \mathrm{sec}$. The earclips were then removed and the subject was returned to the home cage.

CRF(ECS) and VR(ECS). The subjects in these two groups were given one nonreinforced runway trial and the microalligator clips were then attached to the subject's ears. A 500-msec 50-mA ECS was immediately delivered through the earclip electrodes, after which the subject was returned to the home cage.

On the following day, all subjects were given 30 massed extinction trials. During extinction, the stimulus conditions were identical to those during acquisition except that no reinforcement was present in the foodcup in the goalbox.

\section{RESULTS}

The total latencies for each subject were recorded after 10, 20, and 30 extinction trials, and results showed that the shortest latencies were shown by Group VR(ECS), followed in order by Groups VR(NT), CRF(ECS), and CRF(NT). These data are shown in Table 1 . Reciprocals of these data were taken and were subjected to an analysis of variance which showed that the main effect of reinforcement schedule was significant beyond the .05 level; the treatment main effect was not significant, but the Reinforcement Schedule by Treatment interaction was significant beyond the .05 level. The main effect of trials was significant beyond the .05 level, but the Trials by Schedule, Trials by Treatment, and Trials by Treatment by Schedule interactions were not significant at the .05 level.

Groups comparisons, collapsed across trials, showed that the comparisons VR(NT) vs. CRF(NT) and $C R F(E C S)$ vs. CRF(NT) were significant beyond the .05 level, while the comparisons VR(ECS) vs. $\mathrm{VR}(\mathrm{NT})$, and $\mathrm{VR}(\mathrm{ECS})$ vs. $\mathrm{CRF}(\mathrm{ECS})$ were not significant.

Comparisons among groups within trials showed that for the first block of 10 trials, only the comparison VR(NT) vs. CRF(NT) was significant

Table 1

Latencies by Block in Extinction

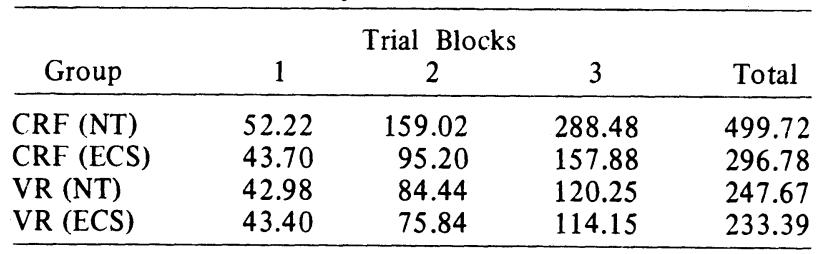

beyond the .05 level. The same comparisons on the second block of 10 trials showed that the comparisons VR(NT) vs. CRF(NT) and CRF(ECS) vs. CRF(NT) were significant beyond the .05 level but the comparison VR(NT) vs. CRF(ECS) was not significant. Comparisons on the third block of 10 trials showed that the comparisons CRF(ECS) vs. $\mathrm{CRF}(\mathrm{NT}), \mathrm{VR}(\mathrm{NT})$ vs. $\mathrm{CRF}(\mathrm{NT})$ were significant beyond the .05 level, but the comparison VR(NT) vs. CRF(ECS) was not significant.

\section{DISCUSSION}

The finding that the $\operatorname{VR}(\mathrm{NT})$ group was significantly more resistant to extinction than was the $\mathrm{CRF}(\mathrm{NT})$ group is the typical partial reinforcement effect (PRE) and was expected.

The most critical finding in this study is that there was no significant difference in resistant to extinction between the CRF(ECS) and VR(NT) and VR(ECS) groups. Thus the effect of ECS on CRF-trained subjects was that of a dramatic increase in resistance to extinction, to the extent that the PRE was eliminated. These results agree with those from previous studies which measured a different (leverpressing) response (i.e., Young \& Day, 1971).

Also in agreement with earlier studies was the finding that although ECS inflated the rate of responding for VR-trained subjects, the effect was slight and did not approach significance. Presumably ECS would affect VR- and CRF-trained subjects in the same manner; however. Young and Costelloe (1974) suggest that a signiticant increase in responding for VR-trained subjects is not found due to a "ceiling" effect-an already high resistance to extinction for these subjects due to the VR training schedule.

These findings. combined with earlier findings, tend to suggest that the actual effect of ECS is the production of hyperactivity in the organism, and this increased drive level, channeled into the response which the subject has learned to make in that stimulus situation, would result in an inflated rate of responding in extinction. These findings also suggest that perhaps the consolidation hypothesis is not the proper interpretation for many studies in which ECS has appeared to produce RA.

\section{REFERENCES}

Dempsey, G. L., \& Young, A. Grant. The effects of electroconvulsive shock on extinction. Bulletin of the Psychonomic Society, 1973, 1, 129-131.

LEwis, D. J. Sources of experimental amnesia. Psychological Review, 1969, 76, 461-472.

McGAUGH, J. L. Time-dependent processes in memory storage Science, 1966, 153, 1351-1358.

Young, A. Grant, \& Costelloe, C. S. The effect of ECS on extinction. Bulletin of the Psychonomic Society, 1974, 3, 133-134.

Young, A. GRANT, \& DAY, H. D. Effect of ECS on one-trial learning and on the partial reinforcement effect. Psychonomic Science, 1971, 24, 99-100.

(Received for publication October 23, 1975.) 